\title{
Area based Mobility Management by using GPS in LEO Satellite Networks
}

\author{
Debabrata sarddar(PhD \\ Scholar) \\ Department of E.T.C.E. \\ Jadavpur University
Suman Kumar Sikdar
M. Tech in C.S.E \\ Jadavpur University \\ Shabnam \\ Bandyopadhyay \\ Department of C.S.E \\ Jadavpur University
}

\author{
Dipsikha Ganguly \\ Department of E.T.C.E. \\ B.P.Poddar I.M.T.,WBUT
}
Sougata Chakraborty
M.Tech in C.S.E
Jadavpur University

Kallyan Kumar Das
M. Tech in C.S.E
Jadavpur University

\author{
Soumya Das \\ Department of E.T.C.E \\ Bengal Institute of tec.WBUT
}

\author{
Kunal Hui \\ M. Tech in C.S.E \\ Haldia Institute of Technology \\ Mrinal Kanti Naskar \\ Department of E.T.C.E \\ Jatavpur University
}

\begin{abstract}
Though Low Earth Orbit (LEO) satellites have some distinct characteristics such as low propagation delay, low power requirements and more efficient spectrum allocation due to frequency reuse between satellite and spotbeams but the higher relative speed than terrestrial mobile networks decreases the quality of service as a result of a huge number of handovers. To overcome this problem a number of handover management schemes have been proposed out of which Mobile IP (MIP) is the standard one. But its mobility management cost is too high. Here we have proposed a low cost area based mobility management method (ABMM) using GPS and Paging for LEO satellite networks, which use GPS to decrease Paging cost and location update cost. Through mathematical analysis simulation results shows that this method is better than the standard mobility management methods.
\end{abstract}

\section{Keywords}

Mobility management, GPS , LEO, spotbeam, ABMM.

\section{INTRODUCTION}

Satellite communication networks are utilized to co-exist with terrestrial networks in order to provide global coverage to a heterogeneously distributed over population. A LEO satellite takes about 100 minutes to orbit the earth, which means that a single satellite is in view of ground equipment for only a few minutes [1]. As a consequence, a LEO satellite system must hand over between satellites to complete the transmission if a transmission takes more than the short time period that any one satellite is in view,. In general, this can be accomplished by constantly relaying signals between the satellite and various ground stations, or by communicating between the satellites themselves using "inter-satellite links" (ISLs) [1], [2].

In addition, LEO systems are designed to have more than one satellite in view from any spot on Earth at any given time, minimizing the possibility that the network will lose the transmission. Because of the fast-flying satellites, LEO systems must incorporate sophisticated tracking and switching equipment to maintain consistent service coverage. The need for complex tracking schemes is minimized, but not obviated, in LEO systems designed to handle only short-burst transmissions [3].

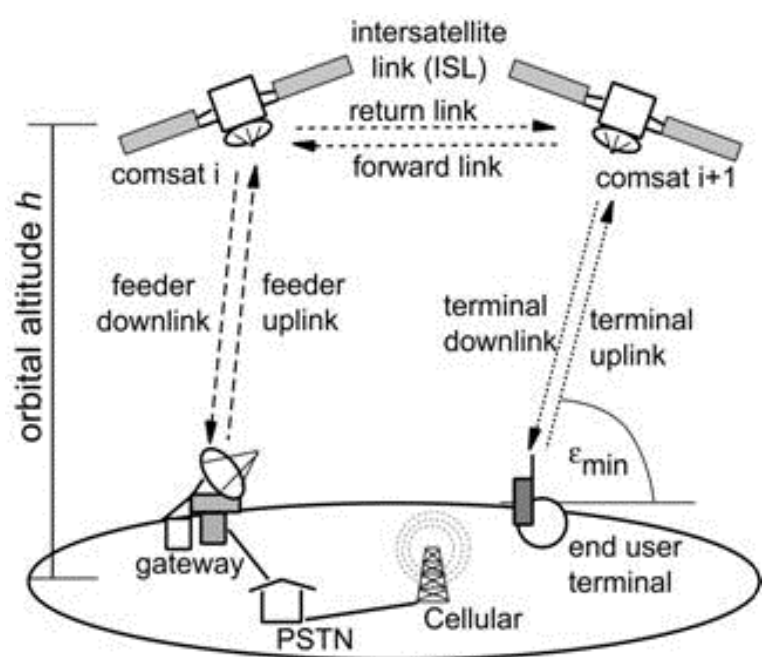

Fig 1: Satellite Communication System

\section{Handover:}

In the term handover or handoff refers to the process of transferring an ongoing call or data session from one channel connected to the core network to another. In satellite communications it is the process of transferring satellite control responsibility from one earth station to another without loss or interruption of service. Handovers may degrade the system performance as an unsuccessful handover results call blocking and forced call termination. Forced call termination is less desirable than a new call blocking though both affect the performance of the system. A number of handover techniques have been proposed to solve this problem.

IP/LEO satellite networks are used to provide a wide variety of IP-based applications, such as teleconferencing and teleeducation. Being totally independent of terrestrial networks, LEO satellite networks have a unique ability of supporting certain emergency communication systems, such as I Am 
Alive (IAA) System [4]. To provide such applications, scalable mobility management and IP communication between end nodes are required.

Conventional IP mobility management protocols, typified by Mobile IP [5] and LIN6 (Location Independent Network architecture for IPv6) [7], require mobile nodes to send binding update requests to the Location Directory every time a handover occurs. Given the high-mobility of satellite networks, usage of these approaches will result in a large number of binding update requests and consequently affect the scalability of the mobility management schemes.

Now we will try to find an equation of rate of handover occurrence form the boundary crossing model.

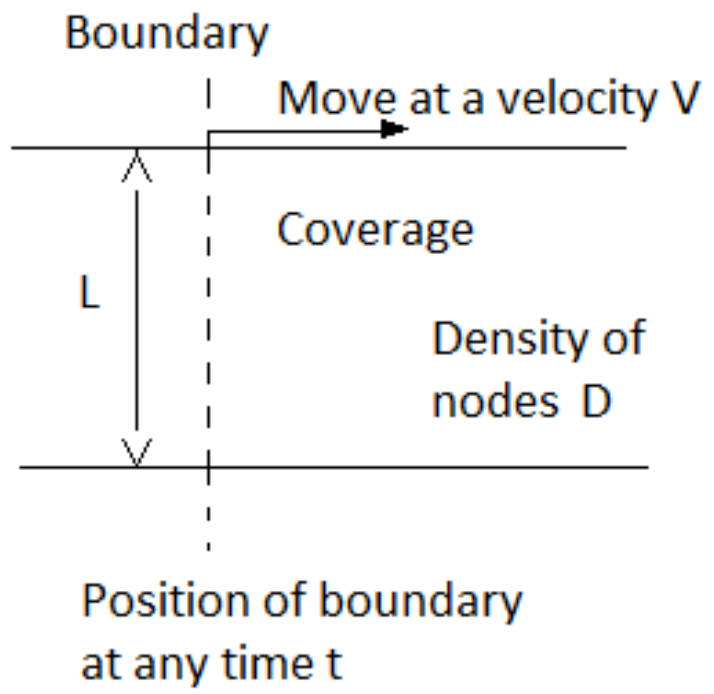

Fig: 2 Boundary crossing model

In the above figure, a coverage boundary of length $\mathrm{L}$ moves with velocity $\mathrm{V}$ from left to right during a period of time $\Delta \mathrm{t}$. The nodes that belong to the area with surface L.V will be required to perform handover during time $\Delta t$. Denoting the area density of nodes as $\mathrm{D}$, the rate of boundary crossing event, $\mathrm{R}$, can be expressed as:

$$
\mathrm{R}=\mathrm{V} . \mathrm{L} \cdot \mathrm{D}
$$

Considering the fact that handovers are mainly due to satellites movement, $V$ can be approximated to the ground speed of satellites. Let $D_{L}\left(V_{\text {sat }} . t\right)$ denote the linear density of nodes on the coverage boundary at time $t$. The rate of handover occurrence, $R_{H O}(t)$, is:

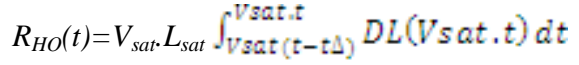

Where, $\mathrm{V}_{\text {sat }}$ and $\mathrm{L}_{\text {sat }}$ denote the ground speed of satellite and the coverage boundary length, respectively.

Since satellites are assumed to cover wide areas and move fast, $V_{\text {sat }}$ and $L_{s a t}$ are large. From Eq.2, it becomes evident that $R_{H O}(t)$ takes large values even for small values of $\Delta t$. Furthermore, this rate of handovers is likely to become even larger in a very populated area (large values of $D_{L}\left(V_{\text {sat }} \cdot t\right)$ ). In this paper we have proposed a new mobility management method named Area Base Mobility Management (ABMM) by using GPS

The rest of the paper is organised as follow: in the second section we have described the related mobility management methods available. In the third section we have described the details of ABMM method. In the forth section we have compared the handover management cost of standard IP protocols our proposed work. The simulation results of related mobility management methods and ABMM method based on handover cost is evaluated in section five. In the next section we conclude the whole paper and finally a future work is mention regarding this paper in section seven.

\section{RELATED WORK}

\subsection{Mobility Management in Terrestrial Mobile Networks and LEO satellite \\ Networks}

\subsubsection{Outline of General Mobility Management:}

In recent years the main concern in IP/LEO networks is the mobility management. The purpose of it is to locate MNs in the network and to guarantee a seamless data transmission upon change in node position. Mobility management mainly deals with two operations, namely binding update and data delivery [8].

- $\quad$ Binding Update: This operation aims to associate Reachability Identity (Reach.ID) and Routing Identity (Route.ID) of each node.

- $\quad$ Reach.ID: It indicates a unique name of the node and not subjected to change.

- Route.ID: It specifies position of the node in the network and changes in response to node movement. When a MN changes its position, the Route.ID changes as well as the old binding update is no longer valid. To update the binding, MNs are requested to send their new Route.ID to the Location Directory (LD) [9].

The main disadvantage of this procedure is when LD is geographically too far from the MNs. As a result, the cost of binding update becomes very expensive especially a high mobility environment such as satellite networks [10]. We all know that a handover is a local process which involves only the MN, the old AR, and the new AR where as binding update is a global process that may affect other network elements in addition to the three adjacent entities. We have two types of Location Management namely Precise Location Management and Loose Location Management.

- Precise Location Management: When Route.ID indicates the position of the MN, so data transmission can be done seamlessly with no further operations. This is called Precise Location Management. In this case the MN requires frequent update of MNs registration even upon a slight movement of nodes. Thus the required update cost can be very huge [11]

- Loose Location Management: When Route.ID is used to indicate the location of MN roughly, an additional operation called paging is done to find the position of MN. But in wide paging areas, the paging cost can be very high which the main disadvantage of it is.

So from this discussion we can conclude that Route.ID plays a very important role on the mobility management cost. More attention should be thus paid to the choice of Route.ID type that suites best mobility management in the underlying network.

\subsubsection{Mobility management in Terrestrial IP network:}

The main drawback of mobility management in terrestrial IP networks is the fact that IP addresses that are originally designed for Route.ID's are also used as Reach.ID's in higher layers. As a result, a MN cannot be identified in the higher layers if its IP address changes at handover occurrence time.

The most useful protocol among existing mobility management is Mobile IP (MIP) which was proposed to tackle this problem. It uses two different IP addresses for two 
different identities of MN. First one is referred as Home address and acts as a Reach.ID and second one is Care of Address and serves as Route.ID. Home Agent plays the role of LD in MIP. In this case, locations of MNs are precisely managed by binding update for every handover occurrence. Another mobility management protocol is LIN6 where LIN6 address are used to refer to the Route.ID of mobile nodes. LIN6 addresses are decided according to the AR that mobile nodes are connected to which is similar to CoA of MIP.

MIP and LIN6 uses a precise location management which necessitates a binding update whenever MN changes its position which is devoid of the condition that the MN is communicating or not. So it is better to use a precise location management to the active nodes. But for the inactive nodes a loose location management is sufficient where the no of binding update frequency can be reduced.

The most dominant loose location mobility management protocols are Paging in Mobile IP (P-MIP) [12] and Cellular IP [13]. Paging is a procedure that allows a wireless system to search for an idle mobile host when there is a message destined to it, such that the mobile user do not need to register its precise location to the system whenever it moves. In P-MIP each paging area is consists of a certain number of ARs in the network. Whenever a packet data is destined to an idle node reaches at one of the AR in a paging area, that AR broadcasts a paging request to all the other ARs that subsequently send paging messages within own coverage areas. When an idle $\mathrm{MN}$ receives a paging request, it becomes active. But that $\mathrm{MN}$ is not required to perform binding update within its own paging area. The MN should only update its binding whenever it crosses the paging area boundary. Thus the frequency of binding update can be reduced.

\subsubsection{Mobility Management in LEO satellite networks:}

The most widely used protocol for mobility management over satellite networks is again Mobile IP (MIP) which is proposed by the Internet Engineering Task Force (IETF) to handle mobility of internet hosts for mobile data communications. It is based on the concept of Home Agent (HA) and Foreign Agent (FA) for routing of packets from one point of attachment to other. It is basically completed by four steps.

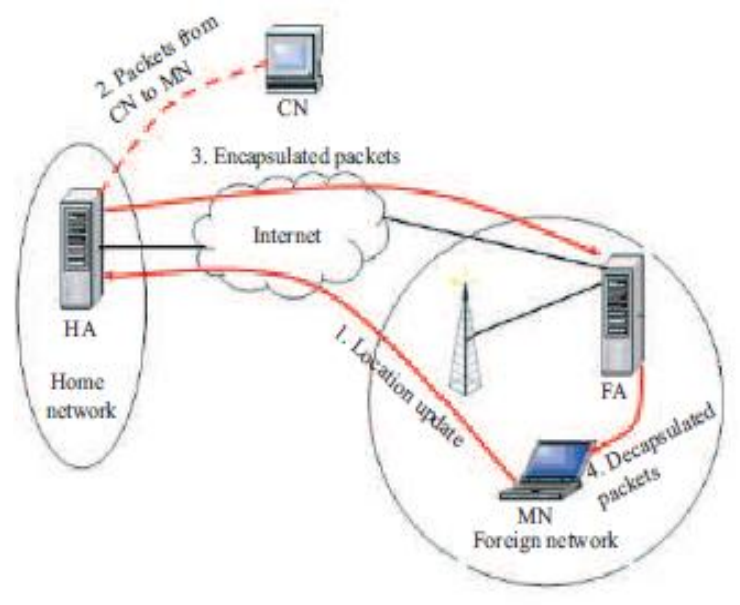

3 Handover Flow in Mobile IP

i) When handover begins MN registers itself in FA and waits for allocation of channels in FA and updates its location in HA directory.

ii) The packets are sent to HA and HA encapsulate it.

iii) Encapsulated packets are sent to The FA. iv) FA decapsulate those packets and sent it to MN.

Applying MIP to LEO satellite networks will result in a precise location management of MNs and consequently an invocation of binding update upon each handover occurrence [14]. As discussed earlier, the number of binding update request will be huge in a single burst. To process all the requests, a massive amount of network bandwidth and computational load are required. This is a serious issue for scalability of mobility management in LEO satellite networks.

To reduce the binding update two loose location management schemes have been introduced; P-MIP and Handover Independent IP Mobility Management [16]. The design of P-MIP encompass, Paging area construction, Movement Detection, Registration, Paging, Data Handling However since paging areas are formed from the coverage areas of a certain number of satellites which are constantly moving, so the ceaseless motion of the satellites makes the paging areas to keep changing. Meanwhile, bursting binding updates might occur as well when LEO satellites cross paging area boundary. So this loose location management method is not suitable for LEO satellite networks.

Another loose location management method is Handover Independent IP Mobility Management which uses the IP addressing on the basis of geographical location and is independent of logical locations.

In this method, the earth's surface is divided into a number of cells, and MN's Route.ID's are associated with the cell where MNs reside in. MNs are assumed to be equipped with GPS (Global Positioning System) receiver for finding their locations. A Route.ID changes and the corresponding binding update occurs only when a MN moves to neighbour cell.

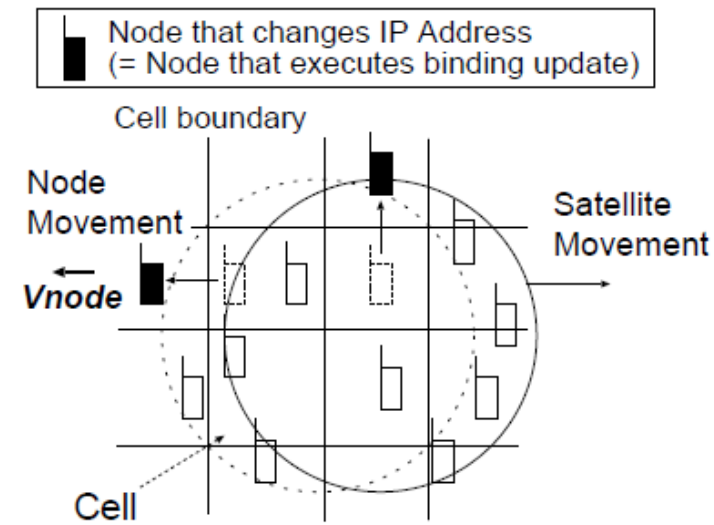

Fig: 4 Handover Independent Mobility

Management

This method has three steps.

1) Geographical Location mapping to Route.ID

2) Cell distribution in a satellite coverage

3) Connection setup and maintenance

The main disadvantages of this method are

$>\quad$ As the LEO satellites have high speed so it crosses the boundaries of the cells frequently which leads to less mitigation of the frequency of IP binding update

$>\quad$ This method needs centralised binding management, which causes huge location directory database and long distance transmission path for IP, address binding updates. This places a heavy burden on communication and storage resources in satellites.

Another mobility management method proposed by Guo Xin et al is named Aviation-oriented Mobility Management method in IP/LEO Satellite networks [17] which considers the 
features of aviation nodes in satellite networks, such as high moving speed, long communication distance, and high connection frequency. The core idea behind the proposal is to associate IP address binding update with ground station coverage areas, decrease the paging cost by introducing location update and cell paging scheme, and set up connections with satellite and ground station forwarding method. In this method, the IP addressing scheme is on the basis of the ground-station real-time coverage areas. The IP addressing scheme is given below

Node IP address $=$ Ground station prefix +

Node ID

Satellite IP address $=$ Ground station prefix + Satellite ID

There are five process involves in this mobility management method: Binding Update, Location Update, Satellite Forwarding, Paging, Ground Station Forwarding. The cost analysis and simulation results show that this method is better than MIP, P-MIP and Handover Independent Mobile IP. But it has the following disadvantages

1. This method does not use a fixed satellite IP address. The satellite IP address prefix changes as it changes the ground station coverage area. As the Speed if satellite is very high, so the satellite changes ground station very frequently. As a result, a huge number of satellite binding update occurs at a time. At the same time, MN binding update request and $\mathrm{MN}$ location update request are also forwarded to ground station. So a huge no of update requests are to be served by ground station which decreases the mobility of the system and increases the cost of the system.

2. In this method if we increase the number of cells in the ground station coverage area, the location update will be more frequent but paging cost will be lower. It will be higher if we increase the cell area by decreasing the no of cells so that the location update will be less frequent. So either the location update cost or the paging cost is higher which increases the total cost.

\section{PROPOSED WORK}

In our proposed work we introduce a new method for Mobility management in LEO networks named Area Based Mobility Management by using GPS in LEO satellite Networks where we have omitted the disadvantages of Aviation-oriented Mobility Management method.

In our proposed method we have used a unique ID for each satellite so that the satellite binding update cost is zero. We have also used GPS to reduce both the location update and paging cost.

In our proposed method, we have divided the total earth surface into on the basis of ground station real time coverage area [19] to reduce the IP address binding update. The mobile nodes are assumed to be equipped with GPS. The IP address of $\mathrm{MN}$ is done as follows

$\mathrm{MN}$ IP address= Ground station prefix $+\mathrm{MN}$ ID

Satellite IP address $=$ Satellite ID

The IP address of satellite is same as the satellite ID because we know that the movement of all satellites is deterministic. So if we have a database of all the satellites with their corresponding coverage areas in every moment if a day so we can find any $\mathrm{MN}$ or ground station is under which satellite coverage area if we know the position of MN.

Here we have shown a sample database using 3astellite and 4 ground station.

\begin{tabular}{|l|ll|ll|ll|}
\hline & GS1 & GS2 & GS3 & \\
\hline Sat 1 & $11-20 \quad$ to & $11-40 \quad$ to & $12-00 \quad$ to \\
& $11-40$ & & $12-00$ & & $12-20$ & \\
& & & & \\
\hline Sat 2 & $11-05 \quad$ to & $11-25 \quad$ to & $11-45 \quad$ to \\
& $11-25$ & & $11-45 \quad$ & $12-05$ & \\
\hline Sat 3 & $10-50 \quad$ to & $11-10$ to & $11-30 \quad$ to \\
& $11-10 \quad$ & & $11-50$ & \\
& & & $11-30$ & & & \\
\hline
\end{tabular}

Here we have assumed each ground station coverage area is server by each satellite by 20 minutes and the overlapped time is $5 \mathrm{~min}$. For every satellite and every ground, this database should be formed.

\section{Ground Station Real time Converge area formation}

The proposed method divides each ground station coverage area (GSCA) into a big centre cell (BCC) and small corresponding cells (SCC). The BCC is further divided into corresponding SCCs. The BCC is used for paging and the SCC (outside BCC) is used for tracking MN by GPS. BCCs and each SCC have unique ID.

In the fig. 5 we have the cell distribution in ground station real time coverage area. Here we have used the hexagonal-shaped cell size. In the figure, GSCA is formed by 19 SCCs, BCC is formed by 7 SCCs, C1-C7 indicated by thick border. We see that one BCC is surrounded by 12 SCC, C8-C19. The area of $\mathrm{BCC}$ is the $36.84 \%$ of the total area

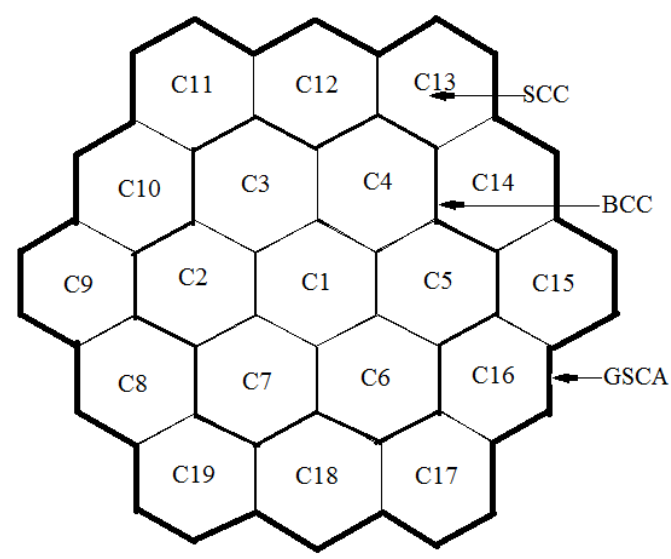

Fig: 5 Cell distributions in ground station real time
coverage area

Location Update and cell paging:

Whenever a MN moves within BCC, it does not have to register its location as it is loosely managed. Whenever a message destined to it, the actual position can be found by sending a paging request within BCC. As the paging request is only sent to the BCC (not total ground station) so the paging cost is less.

When a MN moves from BCC to SCC or one SCC to another SCC or any SCC to BCC it registers its location update to ground station. As approximately $36.84 \%$ coverage area of ground station is covered by BCC so the no of location update is less. The actual position of MN can be found by GPS and then we can find in which SCC it belongs to. Thus we have decreased the location update cost and paging cost simultaneously by using GPS as the cost of GPS is very low compared to location update cost and paging cost. 


\section{Mobility Management process}

As the speed of satellites is very high so the satellites changes the ground station coverage area very frequently. Unlike aviation-oriented mobility management process, here we have used unique satellite ID. Using the database we can easily find the starting and ending time of each coverage area. Also the overlapped timing is also known. Thus whenever a Ground station experiences an overlapped area it re-establishes its connection with the next satellite.

The overall Mobility management process is given in figure 7

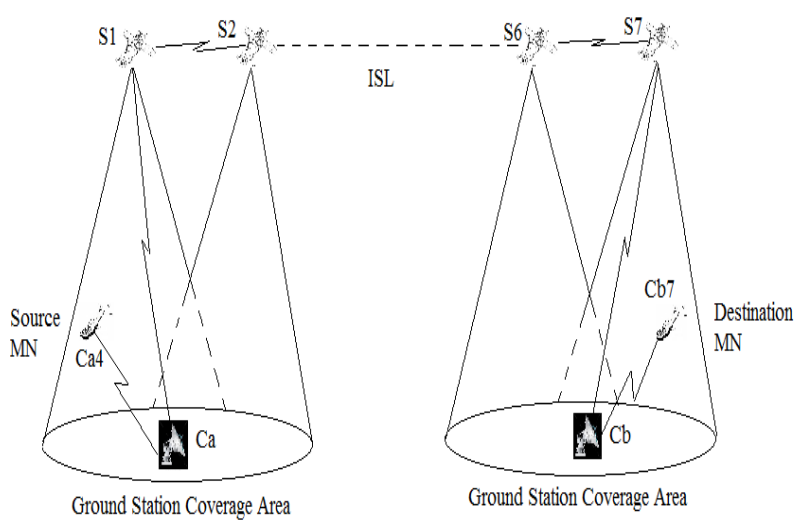

Fig: 6 Connection set up in ABMM method

In figure 6 we have shown how a new call is being established. As shown in the figure, source MN Ca4 (means $\mathrm{MN}$ resides in the $4^{\text {th }}$ cell of GSCA Ca) wants to establish a connection with destination MN Cb7. So MN4 sends a connection request to $\mathrm{Ca}$. Ca forwards this message to satellite S1. S1 now analyses the database and finds that this time destination $\mathrm{MN} \mathrm{Cb} 7$ is being served by Ground station $\mathrm{Cb}$ and $\mathrm{Cb}$ is under the coverage area of satellite S7. Now S1forwards this message to S7 through ISLs. When it reached to Satellite $\mathrm{S} 7$, it forwards this message to GSCA $\mathrm{Cb}$. Now Ground station $\mathrm{Cb}$ analyses from prefix that it is in the BCC. So it spreads the paging request in the all cells of BCC. After receiving the paging request, the connection has been established from $\mathrm{Ca} 4$ to $\mathrm{Cb} 7$.

\section{Advantages of ABMM:}

Our proposed method has following advantages

1. This method reduces the Location Update cost by reducing location update frequency as the location update only requires when $\mathrm{MN}$ crosses the SCCs and SCC to BCC and vice versa.

2. This method also reduces the Paging cost as the paging message is only broadcasts to $\mathrm{BCC}$ not whole ground station coverage area.

3. The IP addresses of satellites are fixed. So the binding update cost of [17] has been reduced.

\section{Cost Analysis:}

In this section we evaluated the cost of proposed method and compared it to MIP, P-MIP, Handover Independent Mobile IP and Aviation oriented mobile IP.

\section{Mobility Management Cost elements:}

As discussed earlier, the mobility management cost mainly consists of the binding update cost and data delivery cost. In precise location management method, the binding update cost is large as a large number of binding update request is generated. In loose location management, this cost is reduced but some additional cost such as local forwarding cost and paging cost are generated. So the overall cost rises.

Mobility Management Cost Definition :
In [12] the mobility management cost is evaluated as the product of generated control message size, $\mathrm{M}$ and the number of hopes, $\mathrm{H}$, required to deliver the message. If we apply such definition into the paging cost, it will be proportional with the number of receivers. Taking into account the broadcasting capabilities of satellites, however, the cost is also simply a product of the message size and the number of travelled hops.

Cost=M.H

Costs of different Mobility management events:

The following defines the cost required for each mobility management event; binding update, local forwarding, paging and GPS finding.

For each case, the Control messages generated are assumed to be equally sized (M) in all the four events. The number of control messages that are generated upon a handover occurrence between mobile nodes and the corresponding ARs, is assumed to be same for MIP, P-MIP, handover Independent Mobile IP and our proposed method. Thus we can neglect the number of control message in the cost evaluation.

Binding Update Cost: Let $H_{M N, L D}$ denote the number of hops between the mobile node and the location directory, $H \mathrm{MN}, \mathrm{MN}$ the number of inter-satellite link hops between the source node and the objective node, and $\alpha$ the ratio of the active mobile nodes to the total number of nodes. The cost of the binding update procedure would be_3M. $\mathrm{H}_{\mathrm{MN}, \mathrm{LD}}+$ M. $\mathrm{H}_{\mathrm{MN}, \mathrm{MN}} \cdot \alpha$

Location Update Cost: Let $H \mathrm{MN}, \mathrm{AR}$ denote the number of hops between mobile node and access router (here is the satellite), and let $H \mathrm{GS}, \mathrm{AR}$ denote the number of hops between ground station and access router, so the cost of the binding update procedure could be expressed as $\mathrm{M}\left(\mathrm{H}_{\mathrm{MN}, \mathrm{AR}}+\mathrm{H}_{\mathrm{GS}, \mathrm{AR}}\right)$. $\mathrm{R}_{\mathrm{CC}}(\mathrm{t})$

Message Transfer cost between GS to MN, and Satellite: The by defining the number of hops between GS to MN and satellite, the total message transfer cost is $\mathrm{H}_{\mathrm{MN}, \mathrm{GS}}$

Paging Cost:_We assume that S satellites broadcast the paging messages to mobile nodes within their coverage areas, the paging cost is $\mathrm{M}\left(\mathrm{H}_{\mathrm{AR}, \mathrm{GS}}+\mathrm{H}_{\mathrm{AR}, \mathrm{MN}}\right)$

GPS Finding Cost: The cost to find a MN by GPS method is GP.

Management Cost of MIP, P-MIP, Handover Independent Mobile IP, Aviation-oriented mobile IP and our proposed method :

The costs of Mobile IP, P-MIP, Handover Independent Mobile IP, Aviation-oriented mobile IP and our proposed method are as follows

A. Mobile IP: The cost of MIP is the product of binding update cost and rate of handover occurrence. So the MIP management cost, $\mathrm{C}_{\text {MIP }}(\mathrm{t})$ can be expressed as

$$
\mathrm{C}_{\mathrm{MIP}}(\mathrm{t})=\mathrm{M} \cdot \mathrm{H}_{\mathrm{MN}, \mathrm{LD}} \cdot \mathrm{R}_{\mathrm{HO}}(\mathrm{t})
$$

B. P-MIP: In P-MIP the active MN supdate their binding upon handover occurrence. The idle nodes perform their binding update only when they cross the paging area boundary. So using equation 1 the rate at which boundary nodes cross the paging area boundary at time $t, R_{p \_a r e a}(t)$ is

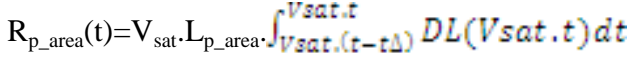


Where $\mathrm{L}_{\mathrm{p} \_ \text {area }}$ denotes the boundary length of paging area.

So the P-MIP cost $\mathrm{C}_{\mathrm{P}-\mathrm{MIP}}(\mathrm{t})$ is

$\mathrm{C}_{\mathrm{P}-\mathrm{MIP}}=\left(3 \mathrm{MH}_{\mathrm{MN}, \mathrm{LD}}+\mathrm{MH}_{\mathrm{MN}, \mathrm{MN} .} \alpha\right) \mathrm{R}_{\mathrm{p} \_ \text {area }}+$

$\mathrm{MH}_{\mathrm{MN}, \mathrm{LD}}\left[\mathrm{R}_{\mathrm{SC}}(\mathrm{t})-\mathrm{R}_{\mathrm{p} \_ \text {area }}(\mathrm{t})\right] \cdot \alpha+$

$\left[\mathrm{MH}_{\mathrm{AR} . \mathrm{AR}}\left(\mathrm{S}_{\mathrm{p}}-1\right)+\mathrm{MS}_{\mathrm{P}}\right] \cdot \mathrm{n}(\mathrm{t})(1-\alpha) \cdot \lambda \quad(6)$

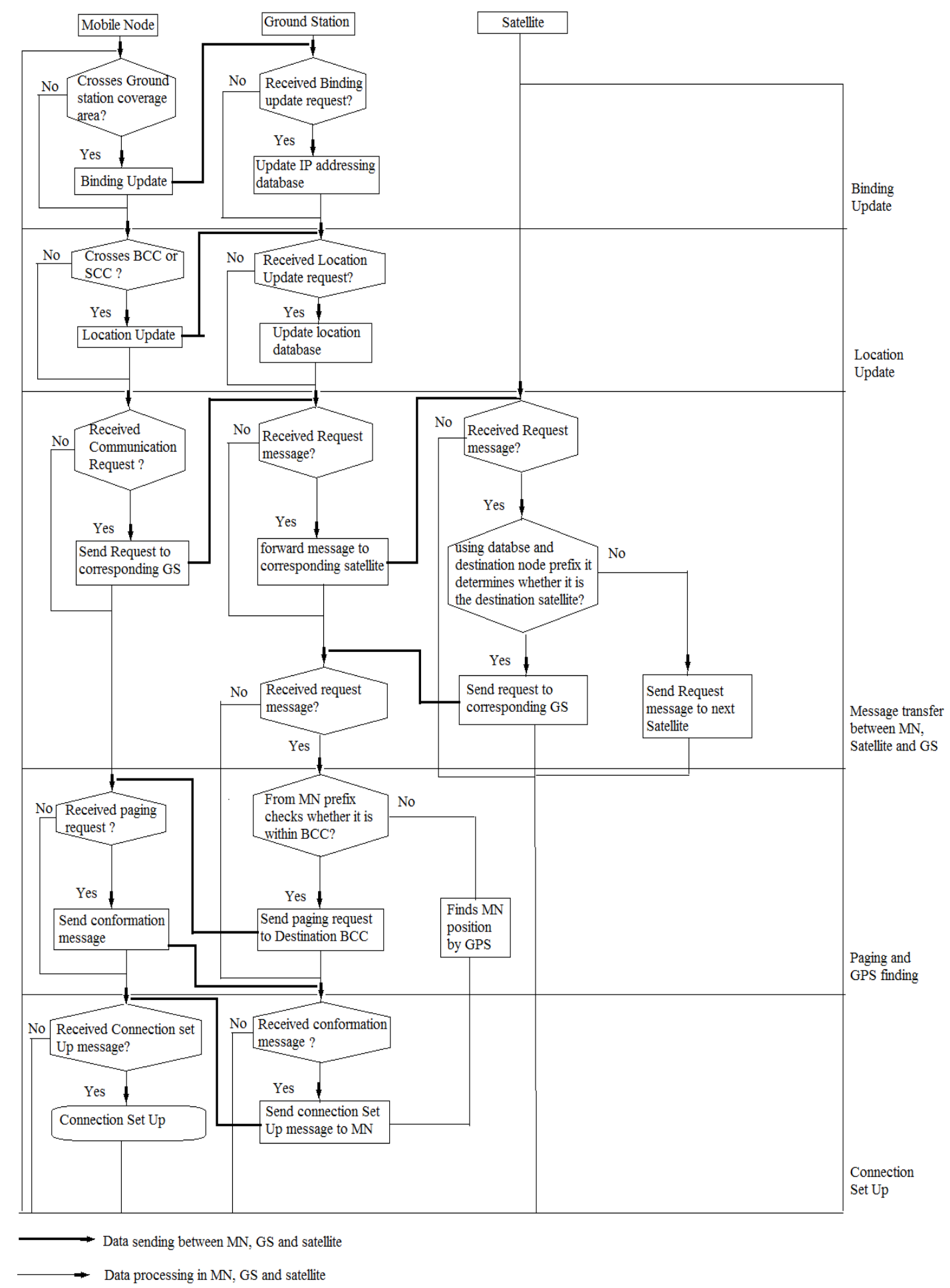

Data sending between MN, GS and satellite

Data processing in MN, GS and satellite
Where $n(t)$ and $\alpha$ denote the total number of nodes per a coverage area at time $t$ and the ratio of active mobile nodes to the total number of nodes, respectively. The rate of newly coming connections to a mobile node is

Fig: 7. Mobility Management in ABMM 
denoted as $\lambda$. The first and second terms indicate the binding update cost, whereas the third one refers to the paging cost. Observe that $n(t) \cdot(1-\alpha) \cdot \lambda$ indicates paging the occurrence rate.

A. Handover Independent Mobility Management Cost: In this method, the local forwarding and paging scheme occurs as some additional cost. So the total $\operatorname{cost} \mathrm{C}_{\mathrm{HI}}(\mathrm{t})$ is

$\mathrm{C}_{\mathrm{HI}}(\mathrm{t})=\left(3 \mathrm{M} \cdot \mathrm{H}_{\mathrm{MN}, \mathrm{LD}}+\mathrm{MH}_{\mathrm{MN}, \mathrm{MN}} \cdot \alpha\right) \mathrm{R}_{\mathrm{cc}}(\mathrm{t})+$ $\mathrm{R}_{\mathrm{SC}}(\mathrm{t}) \cdot \alpha+$

$$
\left\{\mathrm{M} \cdot \mathrm{H}_{\mathrm{AR}, \mathrm{AR}} \cdot\left(2 \mathrm{~S}_{\mathrm{HI}^{-}}-1\right)+\mathrm{M} \cdot \mathrm{S}\right\} \cdot \mathrm{n}(\mathrm{t}) \cdot(1-\alpha) \cdot \lambda
$$

M.HARAR

Where $\mathrm{R}_{\mathrm{CC}}(\mathrm{t})$ can be expressed as

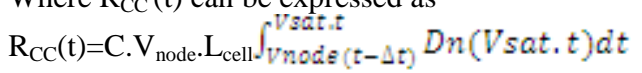

Where $\mathrm{C}$ is the no of cells, $\mathrm{V}_{\text {node }}$ and $\mathrm{L}_{\text {cell }}$ denotes the velocity of nodes and the cell boundary length respectively. The first term in Eq. 7 indicates the binding update cost. The second and third terms represent the local forwarding and paging cost, respectively.

B. Aviation-Oriented Mobile IP: The total cost of Aviation-Oriented Mobile IP is $\mathrm{C}_{\mathrm{GS}}(\mathrm{t})=\left(3 \mathrm{M} \cdot \mathrm{H}_{\mathrm{MN}, \mathrm{LD}}+\mathrm{MH}_{\mathrm{MN}, \mathrm{MN}} \cdot \alpha\right) \cdot \mathrm{R}_{\mathrm{GC}}(\mathrm{t})+$

$$
\begin{array}{ll}
\mathrm{M}\left(\mathrm{H}_{\mathrm{MN}, \mathrm{AR}}+\mathrm{H}_{\mathrm{GS}, \mathrm{AR}}\right) \cdot \mathrm{R}_{\mathrm{CC}}(\mathrm{t})+ & \mathrm{M} \cdot \mathrm{H}_{\mathrm{AR}, \mathrm{GS}} \mathrm{n}(\mathrm{t}) \cdot \lambda+ \\
\mathrm{M}\left(\mathrm{H}_{\mathrm{GS}, \mathrm{AR}}+\mathrm{H}_{\mathrm{AR}, \mathrm{MN}}\right) \mathrm{S}_{\mathrm{GS}} \mathrm{n}(\mathrm{t})(1-\alpha) \cdot \lambda & \text { (9) }
\end{array}
$$

Here

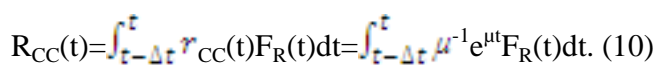

Where $F_{R}(t)$ denotes the linear density of nodes in a boundary of whole coverage area at time $\mathrm{t}$.

C. Proposed Method: In our proposed method, the location update and GPS are used into $63.16 \%$ and Paging is used into $36.84 \%$ of total GSCA. So the total cost of our proposed method is

$\mathrm{C}_{\mathrm{ABMM}}(\mathrm{t})=\left(3 \mathrm{M} \cdot \mathrm{H}_{\mathrm{MN}, \mathrm{LD}}+\mathrm{MH}_{\mathrm{MN}, \mathrm{MN}} \cdot \alpha\right) \cdot \mathrm{R}_{\mathrm{GC}}(\mathrm{t})+$

$\mathrm{M} \cdot \mathrm{H}_{\mathrm{AR}, \mathrm{GS}} \mathrm{n}(\mathrm{t}) \cdot \lambda+\left[\left\{\mathrm{M}\left(\mathrm{H}_{\mathrm{MN}, \mathrm{AR}}+\mathrm{H}_{\mathrm{GS}, \mathrm{AR}}\right) \cdot \mathrm{R}_{\mathrm{CC}}(\mathrm{t})+\right.\right.$

$\left.\mathrm{G}_{\mathrm{PS}}\right\}^{* 63.16}+\mathrm{M}\left(\mathrm{H}_{\mathrm{GS}, \mathrm{AR}}+\mathrm{H}_{\mathrm{AR}, \mathrm{MN}}\right) \mathrm{S}_{\mathrm{GS}} \mathrm{n}(\mathrm{t})(1-$

$\alpha) . \lambda * 36.84] / 100 \quad(11)$

The first term in the equation 11 represents the binding update cost; next term is the message transfer cost between $\mathrm{MN}$ to GS and Satellite. The third term is the summation of Location update cost and GPS finding cost multiplied with the percentage of total area used by both cost and the last term is the paging cost multiplied by the percentage of total area used for paging.

\section{Simulation Results}

In order to evaluate the performance of ABMM method we compare it to MIP, P-MIP, Handover Independent Mobile IP and Aviation-oriented method. Each method is evaluated by handover costs. The simulation results were run on MATLAB 7.8 in a designed virtual environment.

The virtual environment is created by setting the following parameters

\begin{tabular}{|c|l|}
\hline $\begin{array}{l}\text { Satellite coverage area } \\
\text { radius }\end{array}$ & $700[\mathrm{~km}]$ \\
\hline Satellite ground Speed & $7[\mathrm{~km} / \mathrm{sec}]$ \\
\hline Mobile Node speed & $17[\mathrm{~km} / \mathrm{sec}](60 \mathrm{~km} / \mathrm{hr})$ \\
\hline GSCA & $1000 \mathrm{~km}^{2}$ \\
\hline$\alpha$ & \\
\hline
\end{tabular}

\begin{tabular}{|c|c|}
\hline$\lambda$ & 0.0009 \\
\hline $\begin{array}{c}\text { Number of nodes reside } \\
\text { in the coverage area }\end{array}$ & $10^{6}$ \\
\hline$\Delta \mathrm{t}$ & $1 \mathrm{sec}$ \\
\hline $\mathrm{H}_{\mathrm{MN}, \mathrm{LD}}$ & 2 \\
\hline $\mathrm{H}_{\mathrm{MN}, \mathrm{MN}}$ & 1 \\
\hline $\mathrm{H}_{\mathrm{MN}, \mathrm{AR}}$ & 1 \\
\hline $\mathrm{H}_{\mathrm{GS}, \mathrm{AR}}$ & 1 \\
\hline $\mathrm{S}$ & 5 \\
\hline $\mathrm{G}_{\mathrm{ps}}$ & 1 \\
\hline
\end{tabular}

We assume the satellite coverage area to be square shaped and their surfaces are equal to that of a circle with a radius $700[\mathrm{~km}]$. Nodes density is calculated as the ratio of the total number of nodes to the coverage area surface. For the sake of simplicity, effects of cell shapes on the management cost are ignored and cells are assumed to be square shaped.

In Paging Mobile IP, a paging area is constructed by the coverage areas of five satellites that are a certain satellite and its four neighbouring satellites (i.e. $S=5$ ). Each neighbouring satellite is in the same orbit and both adjacent orbits. On the other hands, in the proposed method, $S$ depends on a cell size.

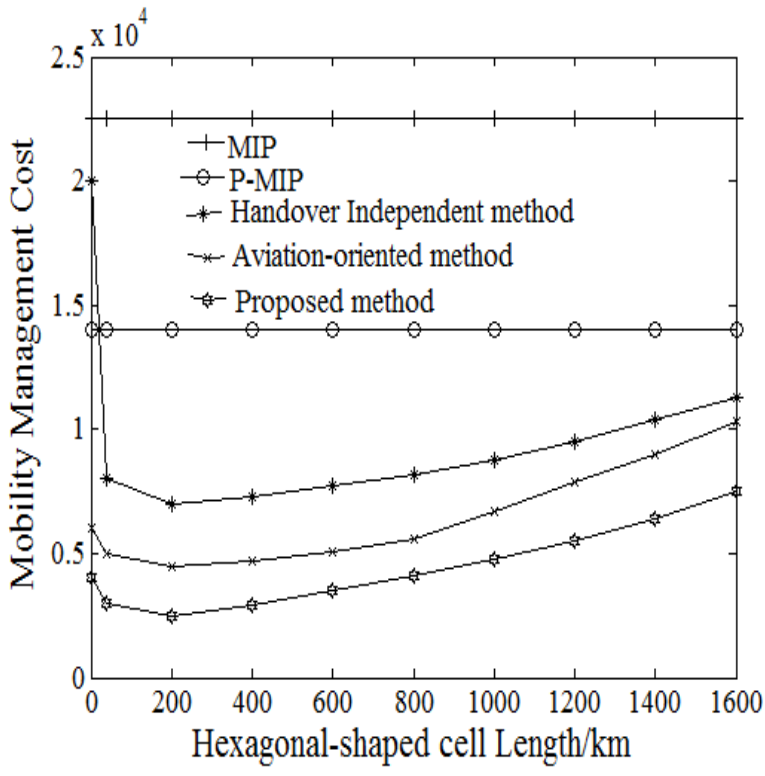

Fig: 8 Mobility Management cost evaluation of five methods

In figure 8 we have shown our simulation results. It shows that our method is better than MIP, P-MIP, Handover independent method, Aviation-oriented method and our proposed method. In comparison with aviation-oriented method, it decreases the location update cost by reducing the frequency of location update. The paging cost is also reduced by decreasing the total paging area. Here we use the GPS 
finding method which introduces additional cost but it is lower in comparison with location update cost and paging cost. So the overall cost decreases.

\section{CONCLUSION}

In this paper, we have proposed the Area Based Mobility Management method in LEO satellite networks where we have reduced the mobility management cost.

We first described what satellite communication is and the advantages of satellite communication. Then we introduce the term 'handover' and the problems of handover on satellite communication. Then we described various IP protocol management methods such as MIP, P-MIP and Handover independent MIP and Aviation-oriented Method. After that we have described our proposed work and mentioned its advantages. The cost analysis of different mobility management methods with comparison with our proposed work is given in the next part of this paper. Based on the cost analysis, a simulation result of these mobility management methods with our proposed works is also given. It shows that the ABMM method is better than other IP protocols. So we can use it in our IP networks as a mode of future satellite communications.

\section{FUTURE WORK}

In future, we will try how to reduce paging cost and location update cost and binding update cost by using GPS.

\section{REFERENCES}

[1] S. L. Kota, P. A. Lippmann, and K. Pahlavan, Broadband Satellite Communications For Internet Access, Kluwer Academic Publishers, 2004.Jamalipour, "Satellites in IP networks," in Wiley Encyclopedia ofTelecommunications, vol. 4, Wiley, 2002, pp. 21112122

[2] L. Strand, "Linux mobile IPv6 HOWTO,” Apr. 2004.

[3] N. Tada, Y. Izawa, M. Kimoto, T. Maruyama, H. Ohno, and M. Nakayama, "IAA system ("I Am Alive"): The experiences of the Internet disaster drills," Proc. INET'00, Internet Society, Jun. 2000.

[4] C. Perkins. "IP mobility support for IPv4," RFC3344, Aug. 2002.

[5] P. K. Chowdhury, M. Atiquzzaman, W. Ivancic ,Handover Schemes in Satellite Networks: State-of-theart and future research directions IEEE communications surveys 4TH Quarter 2006, Vol. 8, NO. 4A.

[6] M. Ishiyama, M. Kunishi, K. Uehara, H. Esaki, and F. Teraoka, "LINA: A new approach to mobility support in wide area networks," IEICE Trans. Commun. E84B(8):2076-2086, 2001.

[7] J. T. Malinen and C.Williams, "Micromobility taxonomy," Internet Draft, IETF, Nov. 2001

[8] M. Atiquzzaman, S. Fu, and W. Ivancic, -TraSH-SN: A transport layer seamless handoff scheme for space networks, $\|$ in Proc. ESTC 2004, Palo Alto, CA, June 2004.

[9] P. Bhagwat, C. Perkins, and S. Tripathi, "Network layer mobility: An architecture and survey," IEEE Pers. Commun., vol. 3, no. 3, pp. 54-64, June 1996.

[10] A. T. Campbell, J. Gomez, S. Kim, Z. Turanyi, C.-Y. Wan, and A. Valko, "Comparison of IP micro-mobility protocols," IEEE Wireless Commun. Mag., vol. 9, no. 1, Feb. 2002.
[11] X. Zhang, J. G. Castellanos, and A. T. Campbell, -Design and performance of mobile IP paging,\| $A C M$ Mobile Networks and Appl., vol. 7, no. 2, Mar.2002.

[12] A. G. Valko, -Cellular IP - a new approach to Internet host mobility, ACM Computer Commun. Rev., Jan. 1999

[13] T"uys"uz and F. Alag"oz, - Satellite mobility pattern based handover management algorithm in LEO satellites,\| in Proc. IEEE ICC 2006, Istanbul,Turkey, June 2006

[14] Debabrata Sarddar, Shubhajeet Chatterjee, Ramesh Jana, Shaik Sahil Babu, Hari Narayan Khan, Utpal Biswas and M.K.Naskar, -Fast Handoff Implementation by using Curve Fitting Equation With Help of GPS\|, International Journal of Computer Science issues (IJCSI) Vol. 08, Issue 03, pp. 535-542, No. 1, May 2011, ISSN (Online): 1694-0814.

[15] H. Tsunoda, K. Ohta, N. Kato, and Y. Nemoto, "Supporting IP/LEO satellite networks by handoverindependent IP mobility management," IEEE J. Select. Areas Commun., vol. 22, no. 2, pp. 300-307, 2004.

[16] Guo Xin, Zhang Jun, Zhang Tao, Ding Yanwen, "Aviation-oriented Mobility management method in IP/LEO satellite Networks", Chinese Journal of Aeronautics.

[17] Ays, eg"ul T"uys"uz and Fatih Alag"oz, -Satellite Mobility Pattern Scheme for centrical and Seamless Handover Management in LEO Satellite Networksl, JOURNAL OF COMMUNICATIONS AND NETWORKS, VOL. 8, NO. 4, DECEMBER 2006.

[18] Gu M, Zhang J. A novel onboard-gateway-based mechanism to improve TCP performance in aeronautical satellite networks. Chinese Journal of Aeronautics 2007; 20(3): 260265.

[19] E. Papapetrou and F.-N. Pavlidou, -QoS handover management in LEO/MEO satellite systems, Wireless Personal Communications, vol. 24, no. 2, pp. 189-204, February 2003.

[20] E. Cayirci and I. F. Akyildiz, "User mobility pattern scheme for location update and paging in wireless systems," IEEE Trans. Mobile Computing, vol. 1, no. 3, pp. 236-247, 2002.

[21] Debabrata Sarddar, Shovan Maity, Arnab Raha, Ramesh Jana, Utpal Biswas, M.K. Naskar -A RSS Based Adaptive Hand-Off Management Scheme In Heterogeneous Networks Received signal strengthll, IJCSI International Journal of Computer Science Issues, Vol. 7, Issue 6, November 2010, pp. 232 - 239

[22] Debabrata Sarddar, Soumya Das, Dipsikha Ganguli, Sougata Chakraborty, Kunal Hui, Kalyan Kumar Das and Mrinal Kanti Naskar. Article: A New Method for Fast and Low Cost Handover in Leo Satellites. International Journal of Computer Applications 37(7):39-45, January 2012. Published by Foundation of Computer Science, New York, USA

[23] Debabrata Sarddar, Soumya Das, Dipsikha Ganguli, Kalyan Kumar Das, Sougata Chakraborty, Kunal Hui and Mrinal Kanti Naskar. Article: A New Method for Controlling Mobility Management Cost of PatHO-LEO satellite and Mobile IP Network. International Journal of Computer Applications 37(7):32-38, January 2012. 
Published by Foundation of Computer Science, New York, USA.

[24] Debabrata Sarddar, Arnab Raha, Tapas Jana, Shovan Maity, Utpal Biswas, and M.K. Naskar, -Reduction of Error in Handoff Initiation Time Calculation for NextGeneration Wireless Systems\| (IJCSE) International Journal on Computer Science and Engineering Vol. 02, No. 06, 2010, 2047-2052.

\section{AUTHORS PROFILE}

Debabrata Sarddar is currently pursuing his $\mathrm{PhD}$ at Jadavpur University. He completed his M.Tech in Computer Science \& Engineering from DAVV, Indore in 2006, and his B.Tech in Computer Science \& Engineering from Regional Engineering College, Durgapur in 2001. His research interest includes wireless and mobile communication.

Dipsikha Ganguly, currently pursuing B.Tech in Electronics \& Communication engineering at B.P.Poddar Institute of Management \& Technology under West Bengal University of Technology. Her research interest includes wireless communication \& satellite communication.

Soumya Das, son of Mr. Subrata Das and Mrs. Swapna Das, currently pursuing his B.Tech in Electronics \& Communication Engg. at Bengal Institute of Technology under West Bengal University of Technology. His research interest includes mobile communication \& satellite communication.

Suman Kumar Sikder completed his B.Tech in CSE from Murshidabad College of Engineering \& Technology and M.Tech in Computer Science \& Engineering from Jadavpur University in 2011. His research interest includes wireless sensor networks and wireless communication systems.

Sougata Chakraborty is presently an employee of IBM (Kolkata). He has completed his B.Tech in IT from Murshidabad College of Engineering and Technology and M.Tech in Computer Science \& Engineering from Jadavpur University in 2011. His research interest includes wireless sensor networks and wireless communication systems.

Kunal Hui is presently an employee of Cognizant (Kolkata). $\mathrm{He}$ has completed his B.Tech in Computer Science \& Engineering from Murshidabad College of Engineering and Technology (2004-2008) and M.Tech in Computer Science \&
Engineering from Haldia Institute of Technology (20092011). His research interest includes wireless sensor networks and wireless communication systems.

Shabnam Bandyopadhyay is presently an employee of Cognizant (Kolkata). She has completed her B.Tech in Electronics and Communication Engineering from Meghnad Saha Institute of Technology and pursuing M.Tech degree in Computer Science \& Engineering at Jadavpur University Her research interest includes wireless sensor networks and wireless communication systems. Email:

Kalyan Kumar Das completed his BE degree in Computer Science and Engineering from REC (NIT), Durgapur in 2002 and M.Tech. in Computer Technology from Jadavpur University in 2011. His research interest includes wireless sensor networks and wireless communication systems. Mrinal Kanti Naskar received his B.Tech. (Hons) and M.Tech degrees from E\&ECE Department, IIT Kharagpur, India in 1987 and 1989 respectively and Ph.D. from Jadavpur University, India in 2006.. He served as a faculty member in NIT, Jamshedpur and NIT, Durgapur during 1991-1996 and 1996-1999 respectively. Currently, he is a professor in the Department of Electronics and Tele-Communication Engineering, Jadavpur University, Kolkata, India where he is in charge of the Advanced Digital and Embedded Systems Lab. His research interests include ad-hoc networks, optical networks, wireless sensor networks, wireless and mobile networks and embedded systems. He is an author/co-author of the several published/accepted articles in WDM optical networking field that include "Adaptive Dynamic Wavelength Routing for WDM Optical Networks" [WOCN,2006], "A Heuristic Solution to SADM minimization for Static Traffic Grooming in WDM uni-directional Ring Networks" [Photonic Network Communication, 2006], "Genetic Evolutionary Approach for Static Traffic Grooming to SONET over WDM Optical Networks" [Computer Communication, Elsevier, 2007], and "Genetic Evolutionary Algorithm for Optimal Allocation of Wavelength Converters in WDM Optical Networks" [Photonic Network Communications,2008]. Fast Handoff Implementation by using Curve Fitting Equation With Help of GPS. 